\title{
Expression of Snail2 in long bone osteosarcomas correlates with tumour malignancy
}

\author{
Amir-Shaya Sharili • Steve Allen • Ken Smith • \\ Judith Hargreaves • Joanna Price • Imelda McGonnell
}

Received: 1 October 2010 /Accepted: 6 December 2010 /Published online: 5 January 2011

(C) The Author(s) 2010. This article is published with open access at Springerlink.com

\begin{abstract}
Snail2 is a marker of malignancy in epithelial tumours; however, in sarcomas, it is not known if this protein is present. Here we examine the expression of Snail2 in one type of sarcoma, osteosarcoma, and explore its relationship to tumour grade, subtype and anatomical location in cases of long bone and cranial bone osteosarcoma. Long bone osteosarcomas typically have a much greater metastatic capability and a poorer prognosis. We
\end{abstract}

Joanna Price and Imelda McGonnell contributed equally to this work.

Electronic supplementary material The online version of this article (doi:10.1007/s13277-010-0146-1) contains supplementary material, which is available to authorized users.

A.-S. Sharili $\cdot$ S. Allen $\cdot$ I. McGonnell $(\bowtie)$

Department of Veterinary Basic Sciences,

The Royal Veterinary College,

Royal College Street, Camden, London NW1 0TU, UK

e-mail: imcgonnell@rvc.ac.uk

A.-S. Sharili

e-mail: asharili@rvc.ac.uk

S. Allen

e-mail: sallen@rvc.ac.uk

K. Smith

Department of Pathology and Infectious Diseases,

Royal Veterinary College,

London, UK

e-mail: ksmith@rvc.co.uk

J. Hargreaves

Abbey Veterinary Services,

Newton Abbot, Devon, UK

e-mail: judith@abbeyvetservices.co.uk

\section{J. Price}

Department of Clinical Veterinary Sciences, University of Bristol, Langford House,

Langford, North Somerset, UK

e-mail: jo.price@bristol.ac.uk find that Snail2 is expressed in the three main subtypes of long bone osteosarcoma — osteoblastic, chondroblastic and fibroblastic. Regression analysis showed that Snail 2 expression was statistically correlated with tumour grade $(p=0.014)$ in all of these subtypes. Snail2 was only expressed in high-grade cranial bone osteosarcomas, suggesting a link between Snail2 expression and metastasis. This is the first time Snail2 has been associated with any sarcoma, and this study shows that Snail2 may be a useful prognostic marker for this disease.

Keywords Osteosarcoma $\cdot$ Snail2 $\cdot$ Runx $2 \cdot$ Prognostic marker

\section{Introduction}

The Snail family of transcription factors, of which there are at least three members, are zinc finger proteins that function as transcriptional repressors [1]. Target genes are recognised by a conserved carboxy-terminal domain that binds a hexanucleotide DNA motif (5'-CACCTG-3') known as the E-box [2]. The functions of Snaill and Snail2, which was originally named Slug, have been best characterised in the developing embryo where they have been shown to regulate epithelial-mesenchymal transitions (EMT).

This function of Snail family members has been most studied in neural crest cells, where Snail 2 was originally defined as a marker of pre-migratory and migratory neural crest [3]. It is now known that it plays a key role in the process of EMT by downregulating E-cadherin, a cell-cell adhesion molecule, therefore allowing the cells to break their cell-cell contacts and become mesenchymal [4]. Interestingly, during embryonic development, Snail2 is also expressed in some mesenchymal tissues. For example, 
during long bone development in the mouse, Snail2 is present in proliferating and hypertrophic chondrocytes and also perichondrial/periosteal cells [5]. Since these tissues are originally derived from mesenchyme, they do not undergo EMT; therefore, it is likely that Snail2 also regulates other processes. Indeed, it has recently been suggested that Snail2 promotes differentiation of osteoblast precursor cells [6].

It is well-known that developmental mechanisms are recapitulated in many types of cancer. This is particularly true for Snail2 signalling which contributes to progression in a range of epithelial tumour types (e.g., breast, lung and ovarian cancers) by regulating the motility and plasticity of cells [7]. In this context, Snail2 is presumed to be a part of the mechanism that switches the tumour cell phenotype from epithelial to mesenchymal in the process of cell invasion with disease progression. There is, however, a diverse group of mesenchymally derived cancers, the sarcomas, which originate from numerous cell types including fibroblasts, chondroblasts and osteoblasts [8]. Since Snail2 is known to be expressed in many of these mesenchymal tissues in the developing embryo, it is possible that its expression may be reactivated in the process of tumourigenesis; however, to the best of our knowledge, there are currently no reports describing its expression or function in mesenchymal tumours.

Osteosarcoma is one type of mesenchymal tumour and is the most prevalent primary bone tumour [9], occurring predominantly in the metaphyseal region of long bones in adolescents [10]. It is a malignant tumour that often metastasizes to other organs, with a predilection for the lung. Although relatively rare in humans, with one to three cases per million annually in the USA [10], it is much more common in larger breeds of middle-aged dogs with about 8,000 cases diagnosed each year [9]. Clinically canine and human osteosarcomas are indistinguishable with regard to histology and gene expression, and in both cases, the primary tumour tends to develop in the appendicular skeleton and is associated with a high risk of metastasis to the lungs [11]. It has therefore been proposed that these naturally occurring canine osteosarcomas could be used as an appropriate model for the human disease due to these similarities in tumour biology and metastatic behaviour [12] coupled with the easier access to clinical material.

We therefore undertook a study using archived canine material to determine whether Snail2 is re-expressed in osteosarcomas, of various histological grades and subtypes, from different anatomical locations. Furthermore, to determine whether Snail2 expression is common to tumours from other species, we also characterised Snail2 expression in feline appendicular osteosarcomas. Our finding that expression of Snail2 protein is common to both canine and feline osteosarcomas and the very high genetic similarity between canine and human osteosarcomas raises the potential that Snail2 may also be present in human cases.

\section{Methods and materials}

Tissue samples

The tissues used in this study were archived material from a commercial veterinary diagnostic laboratory. All tissues were fixed, processed and embedded in paraffin wax using routine methods. Tissues were sectioned $(4 \mu \mathrm{m})$ and mounted on poly-L-lysine slides for haemotoxylin and eosin staining and immunohistochemical analysis.

\section{Tumour grading}

Osteosarcomas were classified as described by the World Health Organisation (WHO; [13]), being divided into osteoblastic, fibroblastic and chondroblastic types on the basis of the extracellular matrix produced by the neoplastic cells. Tumours were then graded as well-differentiated, intermediate grade and poorly differentiated using standard WHO criteria relating to nuclear pleomorphism, cellular atypia and mitotic index.

\section{Immunohistochemistry}

Sections were de-waxed in xylene, rehydrated in a graded series of ethanols and then washed in distilled water $\left(\mathrm{dH}_{2} \mathrm{O}\right)$ and phosphate-buffered saline (PBS). Endogenous peroxidase activity was quenched by incubation with $3 \%$ hydrogen peroxide in methanol for $30 \mathrm{~min}$. Sections were then pre-blocked with PBS containing $0.05 \%$ Triton X-100 and $10 \%$ calf serum (PBS/TX-100/CS) for $45 \mathrm{~min}$ and incubated with primary antibody overnight at $4{ }^{\circ} \mathrm{C}$. Primary antibodies used were rabbit anti-Snail $2(4 \mu \mathrm{g} / \mathrm{ml}$; Santa Cruz, Inc., USA), rabbit anti-Snail1 $(4 \mu \mathrm{g} / \mathrm{ml}$; Santa Cruz, Inc., USA) and goat anti-Runx2 (4 $\mu \mathrm{g} / \mathrm{ml}$; Santa Cruz). For negative controls, either rabbit or goat IgG was substituted for the primary antibody. The sections were then incubated with either rabbit or goat biotin conjugated secondary antibody (Dako, Glostrup, Denmark) for $45 \mathrm{~min}$. Detection was carried out using the Vector ABC method with 3.3'diaminobenzidine tetrachloride as substrate (Vector Labs, Peterborough, UK). After staining, sections were counterstained with $1 \%$ haematoxylin solution for $1 \mathrm{~min}$, dehydrated in a series of graded ethanols, cleared with xylene and mounted using VectaMount (Vector Labs). Sections were viewed using a Leica DM4000B light microscope and photographed with a DC 500 Leica digital camera (Leica, Solms, Germany). 


\section{Cell culture}

Human osteosarcoma cell line TE85/HOS, rat osteosarcoma cell line ROS 17/2.8, canine osteosarcoma cell line D-17 and human embryonic kidney cells HEK293 were seeded in media (Dulbecco's modified Eagle's medium supplemented with $10 \%(v / v)$ foetal calf serum, $2 \mathrm{mM}$ L-glutamine, and $100 \mathrm{U} / \mathrm{ml}$ penicillin and $100 \mu \mathrm{g} / \mathrm{ml}$ streptomycin) at a density of $5 \times 10^{4}$ cells onto 12 -well plates and incubated at $37^{\circ} \mathrm{C}$ with $5 \% \mathrm{CO}_{2}$ in a humidified atmosphere. When confluent, cells were lysed for Western blotting (refer to Supplemental Fig. 1).

\section{Western blotting}

The specificity of Snail2 antibody was verified using Western blotting in various human, rodent and canine osteosarcoma cell lines and in Snail2 overexpressing HEK293 cells. Immunoblotting was performed as previously described [14]. Briefly, cells were washed in icecold PBS and lysed in denaturing lysis buffer. Genomic DNA was sheared by passage through a Qiashredder column (Qiagen) and proteins and DNA denatured by boiling for $5 \mathrm{~min}$. Protein concentrations were determined by the BCA assay (Perbio, Chester, UK). Ten micrograms of protein was resolved using SDA-PAGE and electro- transferred onto nitrocellulose membranes (Schliecher and Schuell, Dassel, Germany). Membranes were blocked for $1 \mathrm{~h}$ in $5 \%$ BSA before being incubated with primary and secondary antibodies. Proteins were visualized using the enhanced chemiluminescence detection system (GE Healthcare, Amersham Biosciences). Refer to Supplemental Fig. 1.

\section{Immunofluorescence}

Sections were de-waxed, rehydrated and placed in permeabilisation buffer (20 mM Hepes, $300 \mathrm{mM}$ sucrose, $50 \mathrm{mM} \mathrm{NaCl}, 0.5 \%$ TX-100, $3 \mathrm{mM} \mathrm{MgCl}_{2}, 0.05 \%$ sodium azide, $\mathrm{pH}$ 7.0) for $15 \mathrm{~min}$. Non-specific binding was blocked by incubation with wash buffer $(10 \%$ calf serum, $0.05 \%$ sodium azide in PBS) for $1 \mathrm{~h}$ at room temperature. Samples were then incubated with goat antiRunx2 (as described above) overnight at $4^{\circ} \mathrm{C}$. Sections were then washed with wash buffer and incubated with Alexa Fluor ${ }^{\circledR} 594$ donkey anti-goat IgG (Invitrogen Molecular Probes, UK) for overnight at $4^{\circ} \mathrm{C}$ with rabbit anti-Snail2 $\operatorname{IgG}$ followed by Alexa Fluor ${ }^{\circledR} 488$ goat antirabbit IgG (Invitrogen Molecular Probes, UK). Imaging was performed with a Zeiss LSM 510 laser scanning confocal microscope (Carl Zeiss, Inc., Thornwood, NY, USA).

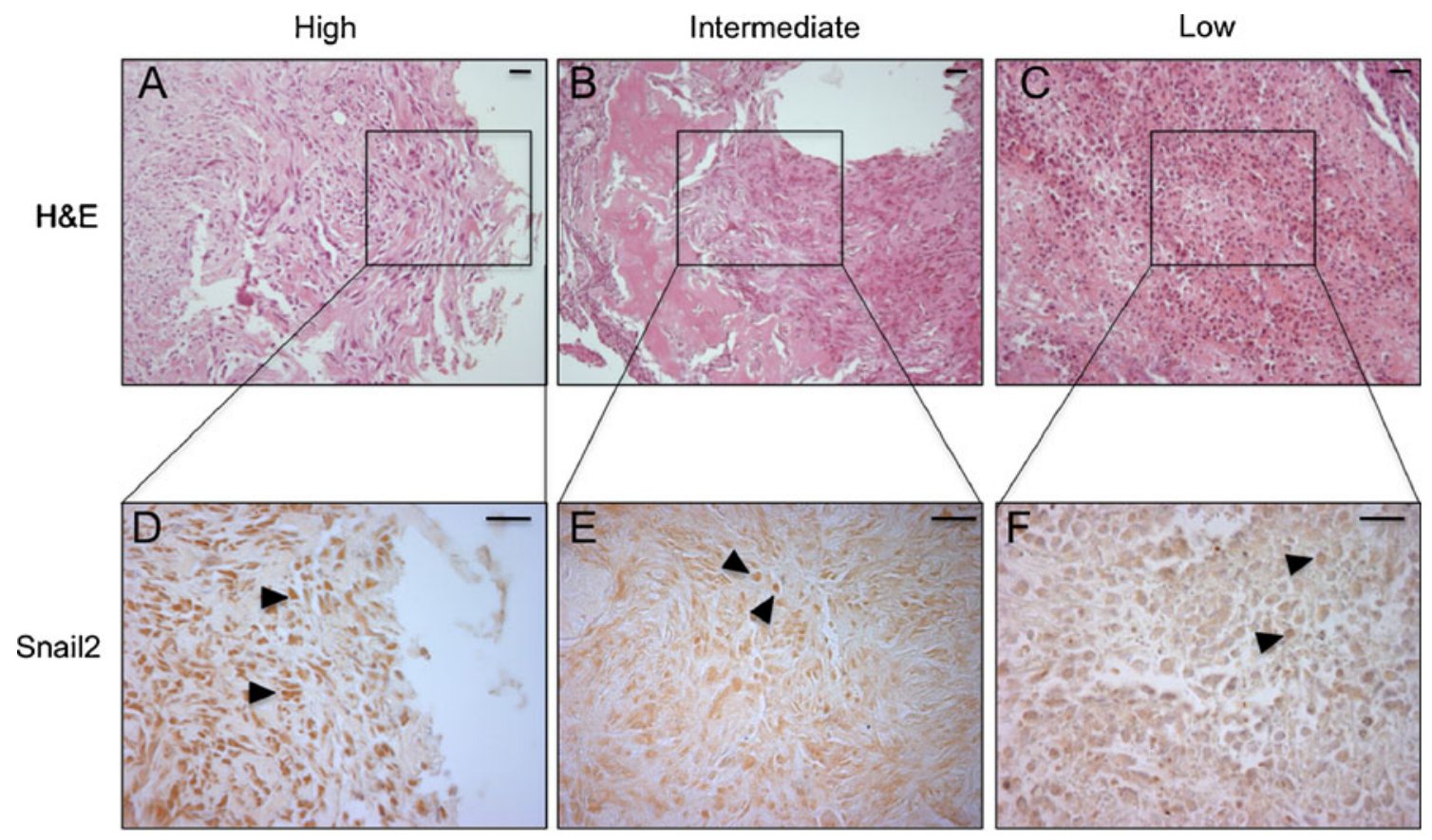

Fig. 1 Histological features and Snail2 expression in various canine osteosarcoma grades. H\&E $(A-C)$ and Snail2 $(D-F)$ in section of osteosarcoma. High-grade $(A, D)$, intermediate-grade $(B, E)$ and lowgrade $(C, F)$ tumours. Boxed area shows regions used for Snail2 localisation. $A$ High-grade tumour showing pleomorphic cellularity with sparse osteoid. $B$ Intermediate-grade tumour with pleomorphic cellularity and osteoid production. $C$ Low-grade tumour showing uniform cellularity and abundant osteoid production. $D$ Intense nuclear Snail2 localisation (arrowheads). E Moderate nuclear Snail2 localisation (arrowheads). $F$ Weak nuclear Snail2 localisation (arrowheads). Scale bars $=10 \mu \mathrm{m}$ 
Statistical analysis

An ordinal logistic regression model was used to assess the association between immunohistochemical expression and tumour grade. Statistics presented are odds ratios (OR) and their $95 \%$ confidence intervals (CI). Kendall's tau- $b$ was presented when there is quasi-separation in the model. Results were considered statistically significant at $p<0.05$, unless otherwise specified. SPSS 17.0 for Windows was used for all analyses.

\section{Results}

Grades of canine osteosarcoma, histological features and Snail2 expression

Osteosarcoma cases in this study were graded from high grade (poorly differentiated) to low grade (well differentiated; Fig. 1 A-C), where high grade is associated with a more aggressive phenotype [15]. Of the 20 cases of long bone osteosarcoma examined, 11 were high-grade, five were intermediate-grade and four were low-grade osteosarcomas (Table 1). Histological examination of high-grade osteosarcoma tissue revealed a highly cellular tumour mass with pleomorphic neoplastic cells surrounded by sparse areas of bony matrix (osteoid; Fig. 1 A). In contrast, low- grade osteosarcomas had a more uniform cellular morphology with more organised osteoid and tumour bone (Fig. $1 \mathrm{C}$ ). In high-grade tumours, Snail2 expression was prominent and largely nuclear (Fig. 1 D), compared with intermediate- and low-grade cases where expression decreased progressively (Fig. 1 E, F). In contrast, Snail1 had a very limited expression that did not correlate with tumour grade (Supp. Fig 2).

Histopathological subtypes of canine osteosarcoma and their expression of Snail2

Classically, canine osteosarcoma is characterised by the presence of spindle-shaped cells producing osteoid [9] (Fig. 1 B). However, several histological subtypes are identifiable depending on the cellular differentiation and type of matrix produced (osteoid, cartilage or fibrous tissue) [10]. In humans, osteoblastic osteosarcomas account for approximately $70 \%$ of cases, while chondroblastic and fibroblastic tumours occur at roughly $10 \%$ each [16]. The remaining $10 \%$ include telangiectatic (vascular) and giant cell osteosarcoma. In this study, histological examination of 20 canine appendicular skeleton osteosarcomas revealed that osteoblastic (15 cases, $75 \%$ ) were most prominent, followed by chondroblastic (three cases, $15 \%$ ) and fibroblastic (two cases, 10\%; Fig. 2 A, D, G; Table 1). In all histopathological subtypes of high-grade osteosarcoma,
Table 1 Summary of clinical and histology data from canine long bone osteosarcoma
$O B$ osteoblastic, $C B$ chondroblastic, $F B$ fibroblastic

\begin{tabular}{|c|c|c|c|c|c|c|}
\hline $\begin{array}{l}\text { Case } \\
\text { no. }\end{array}$ & $\begin{array}{l}\text { Age (year, } \\
\text { month) }\end{array}$ & Sex & Breed & $\begin{array}{l}\text { Tumour } \\
\text { location }\end{array}$ & $\begin{array}{l}\text { Histology } \\
\text { subtype }\end{array}$ & $\begin{array}{l}\text { Histology } \\
\text { grade }\end{array}$ \\
\hline 1 & 7,10 & $\mathrm{~F}$ & Rottweiler & Proximal tibia & $\mathrm{OB}$ & Intermediate \\
\hline 2 & 4,0 & $\mathrm{~F}$ & Rottweiler & Proximal tibia & OB & High grade \\
\hline 3 & 4,0 & M & Labrador & Proximal tibia & OB & High grade \\
\hline 4 & 7,0 & M & Greyhound & Distal radius & OB & High grade \\
\hline 5 & 8,5 & M & Rottweiler & Proximal tibia & $\mathrm{CB}$ & High grade \\
\hline 6 & 8,0 & M & WHWT & Radius & FB & High grade \\
\hline 7 & 8,5 & M & Retriever & Proximal tibia & OB & Intermediate \\
\hline 8 & 9,7 & M & SBT & Radius & OB & High grade \\
\hline 9 & 10,0 & $\mathrm{~F}$ & Labrador & Proximal tibia & OB & Low grade \\
\hline 10 & 1,7 & M & Staffie cross & Proximal tibia & $\mathrm{CB}$ & Low grade \\
\hline 11 & 1,6 & M & Labrador & Proximal tibia & OB & High grade \\
\hline 12 & 8,0 & $\mathrm{~F}$ & Weimaraner & Distal femur & OB & High grade \\
\hline 13 & 11,0 & $\mathrm{~F}$ & Lurcher & Distal femur & OB & High grade \\
\hline 14 & 6,6 & M & Greyhound & Distal radius & OB & Intermediate \\
\hline 15 & 9,0 & M & Rottwieler & Distal radius & OB & Intermediate \\
\hline 16 & 2,0 & $\mathrm{~F}$ & Rottwieler & Proximal fibula & $\mathrm{CB}$ & High grade \\
\hline 17 & 12,0 & M & Retriever & Proximal humerus & $\mathrm{OB}$ & Low grade \\
\hline 18 & 2,6 & $\mathrm{~F}$ & Malamute & Proximal tibia & OB & Intermediate \\
\hline 19 & 10,11 & $\mathrm{~F}$ & GSD & Tibia & FB & Low grade \\
\hline 20 & 8,0 & M & Labrador & Proximal tibia & OB & High grade \\
\hline
\end{tabular}




\section{Snail 2}

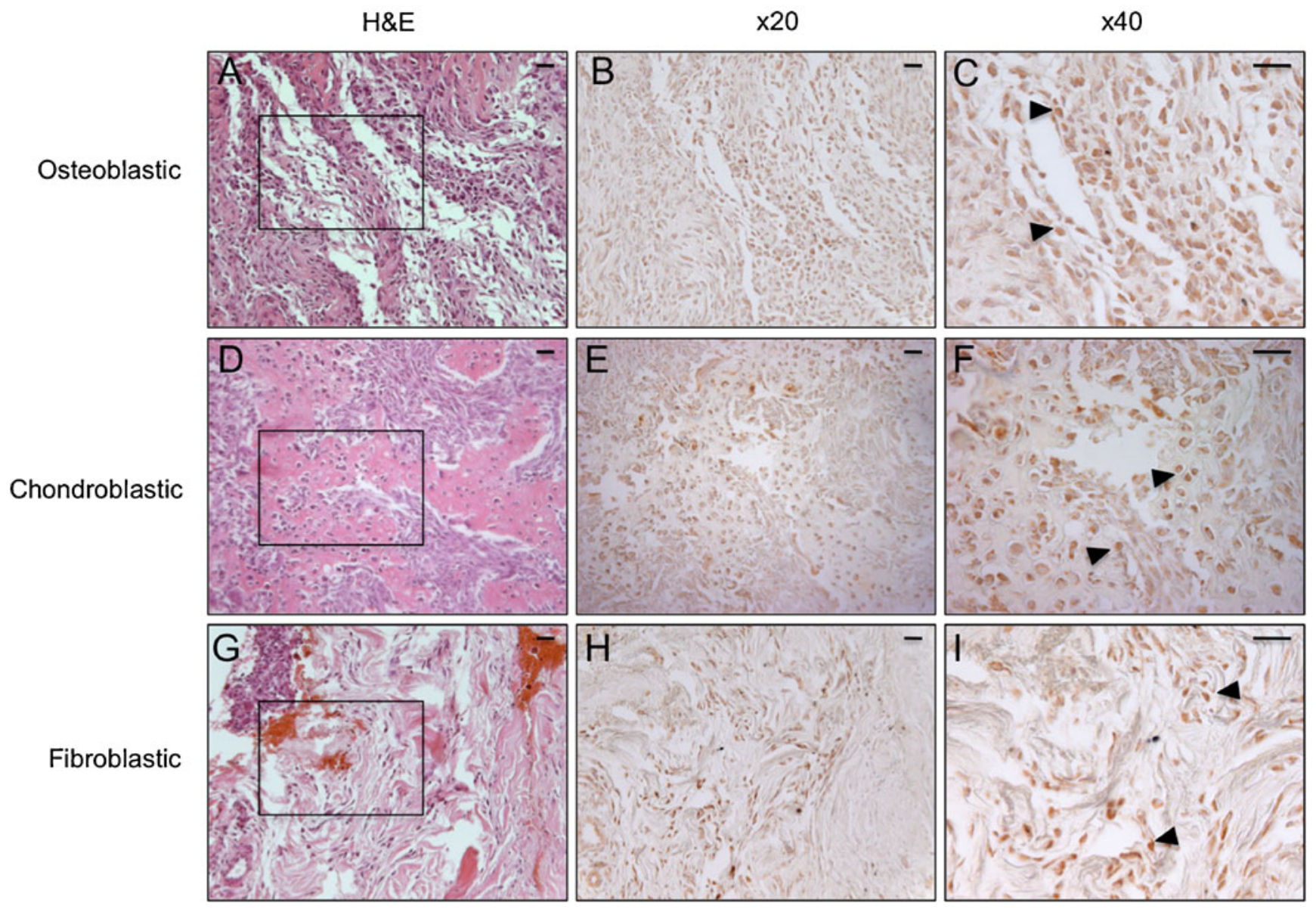

Fig. 2 Snail2 expression in canine long bone osteosarcoma subtypes. $\mathrm{H} \& \mathrm{E}(A, D, G)$ and Snail2 immunolocalisation $(B, C, E, F, H, I)$. Sections of high-grade osteosarcoma showing osteoblastic $(A-C)$, chondroblastic $(D-F)$ and fibroblastic $(G-I)$ subtypes. Boxed area shows regions used for Snail2 immunolocalisation. All high-grade osteosarcomas show Snail2 nuclear localisation (arrowheads in $C, F$, and $I$ ). Scale bars $=10 \mu \mathrm{m}$
Snail2 protein was present (Fig. 2 C, F, I). The initial observation that low-grade tumours had less Snail2 protein also holds true for all three subtypes.

Canine osteoblastic subtype osteosarcomas express the preosteoblast marker Runx2

Osteoblastic osteosarcomas are the most common histological subtype, and they display characteristics of undifferentiated osteoblasts [16]. Therefore to further demonstrate that the canine osteosarcomas in this study had osteoblastic characteristics, we looked for expression of Runx2, a transcriptional regulator necessary for the commitment of mesenchymal cells into the osteoblastic lineage [17]. This analysis showed nuclear Runx2 immunoreactivity in the osteoblastic subtypes (Fig. 3 B), but not in either the fibroblastic or chondroblastic subtypes that we examined (Fig. 3 E, F, H, I). Thus, Runx2 staining shows that high-grade osteoblastic osteosarcomas contain large numbers of preosteoblast-like cells.

Snail2 and Runx2 co-localise in high-grade tibial canine osteosarcomas

Since these canine osteosarcomas expressed both Snail2 and Runx2, we assessed whether these molecules colocalised in cells of 11 high-grade tibial osteosarcomas. Both Snail2 and Runx2 were present in the nuclei of cells in high-grade neoplasm $(n=11 / 11$; Fig. $4 \mathrm{~B}-\mathrm{D}$, $\mathrm{F}-\mathrm{H})$. The mainly nuclear localisation of Snail2 therefore suggests that it is active in the early osteoblastic cells in these tumours [18].

Similar analysis in low-grade tumours showed barely detectable levels of Snail2 and an absence of Runx2 (Fig. 4 $\mathrm{J}, \mathrm{K}, \mathrm{L}$ ). This result suggests that Snail2 is localised in preosteoblast-like cells in high-grade tumours. 


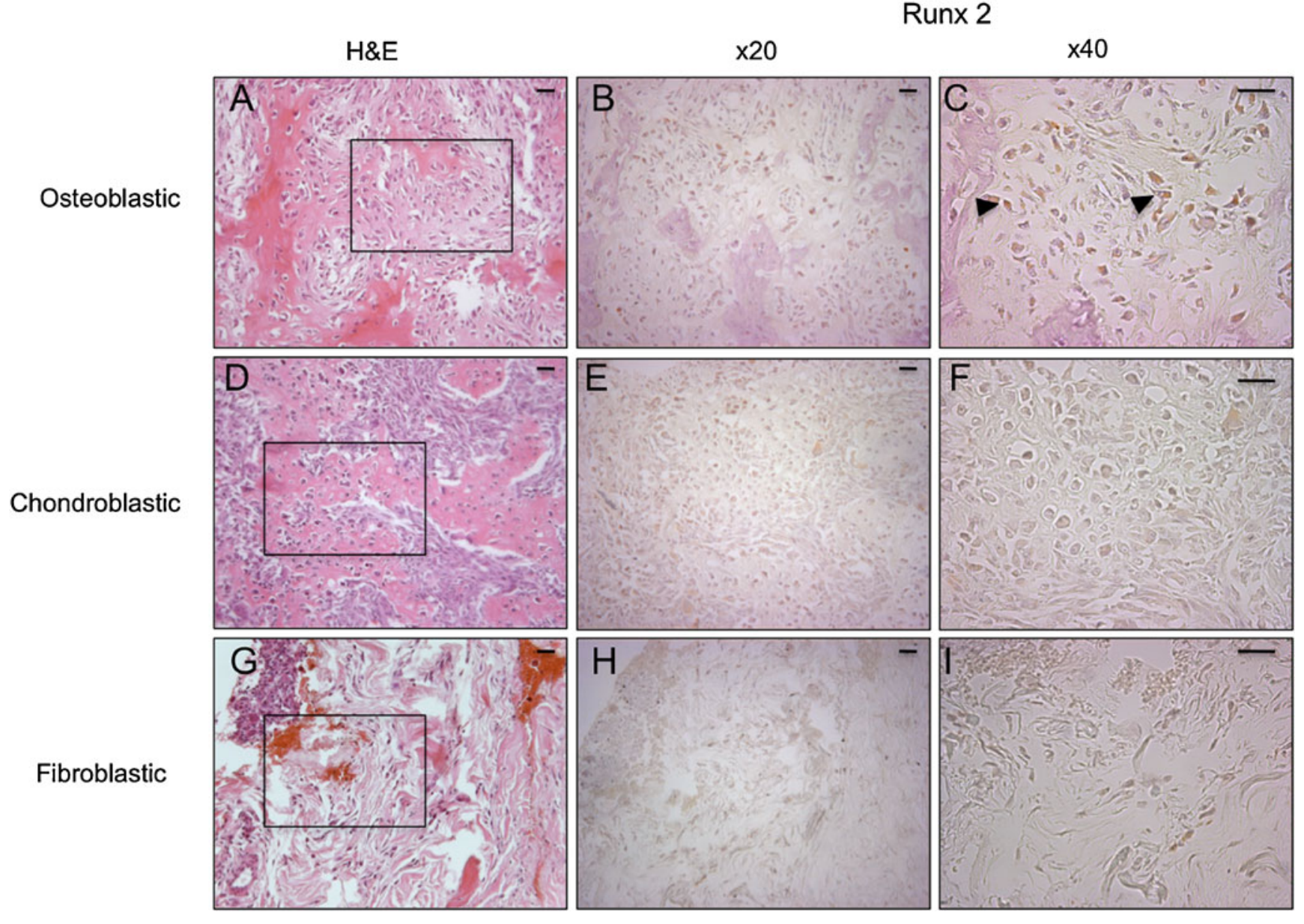

Fig. 3 Runx2 expression in canine long bone osteosarcoma subtypes. $\mathrm{H} \& \mathrm{E}(A, D, G)$ and Runx2 immunolocalisation $(B, C, E, F, H, I)$. Sections of high-grade osteosarcoma showing osteoblastic $(A-C)$, chondroblastic $(D-F)$ and fibroblastic $(G-I)$ subtypes. Boxed area

Localisation of Snail2 and Runx2 in canine osteosarcomas from cranial skeleton

Osteosarcomas of cranial bones are less common than tumours of appendicular bones, and in canines, there is usually a better clinical outcome with only $10 \%$ of tumours undergoing metastasis, in comparison to osteosarcomas of the appendicular skeleton in which $75-85 \%$ undergo metastasis [19]. Having determined the localisation of Snail2 protein in osteosarcomas of the appendicular skeleton, we went on to characterise Snail2 localisation in ten osteosarcomas from flat bones of the cranial skeleton (see Table 2). We found intense Snail2 staining in highgrade cranial skeletal osteosarcomas (Fig. $5 \mathrm{~B}, \mathrm{C}$ ), and this co-localised with Runx2 in the nuclei of cells in these tumours (Fig. 5 D, E).

In low-grade osteosarcomas, Snail2 was barely detectable and Runx2 was absent (Fig. 5 G-J). Interestingly, Snail2 was detected in lower numbers of osteosarcoma cases of the cranial skeleton $(n=4 / 10)$ in comparison with shows regions used for Runx2 immunolocalisation. Only osteoblastic osteosarcomas show positive immunoreactivity (arrowheads in $C$ ). Scale bar $=10 \mu \mathrm{m}$

long bone tumours $(n=20 / 20)$. This suggests that Snail2 is expressed in a higher proportion of tumours of the appendicular skeleton than in the cranial counterparts.

Localisation of Snail2 and Runx2 in feline appendicular osteosarcomas

Osteosarcomas of the appendicular skeleton behave differently in felines compared to canines where they tend to be metastatically more aggressive [20]. We therefore compared the cellular pattern of immunolocalisation of Snail2 in feline versus canine appendicular osteosarcomas. In feline osteosarcomas (see Table 3), a similar pattern of immunolocalisation to that seen in canine osteosarcomas was found. Prominent Snail2 immunoreactivity was detected in high-grade tumours (Fig. 6 $\mathrm{B}, \mathrm{C})$ compared to barely detectable staining in the lowgrade tumours (Fig. 6 G, I). Similarly Runx2 protein was also present in the cells of high-grade tumours (Fig. 6 D) but absent in low-grade tumours. This proves there is a 

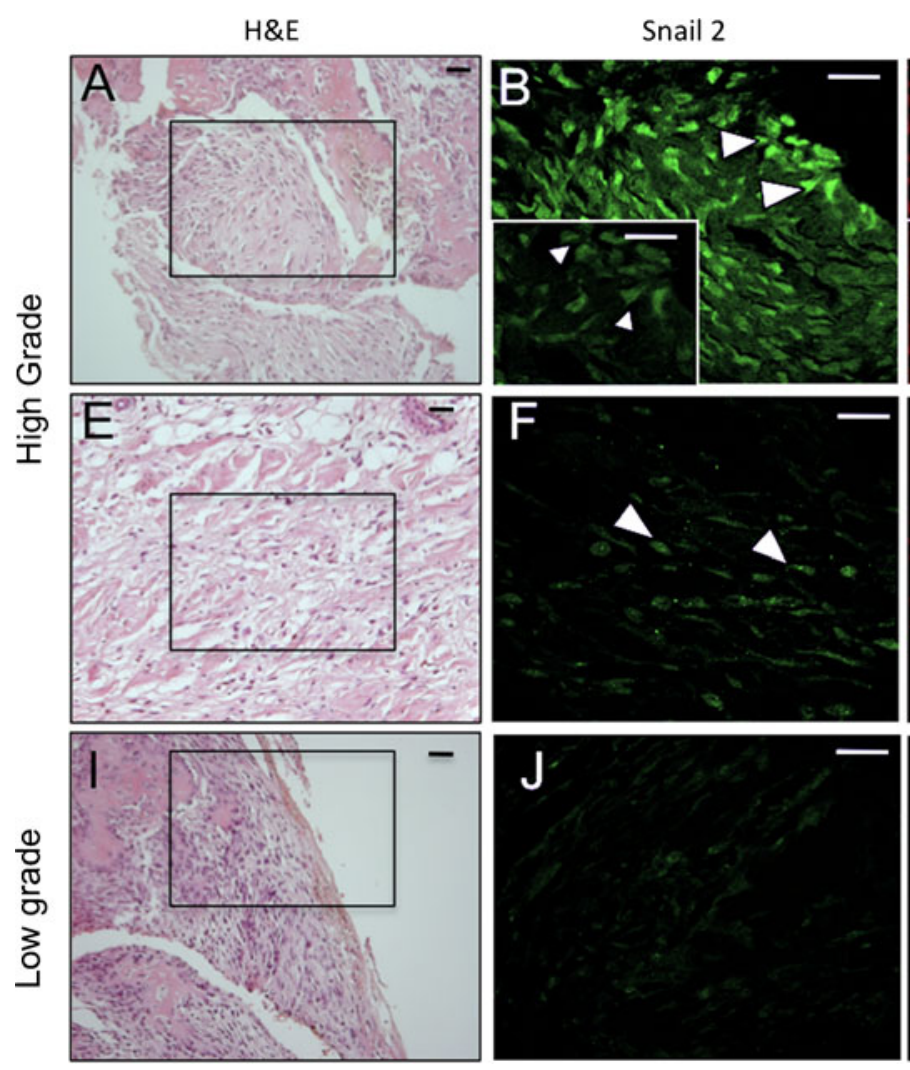
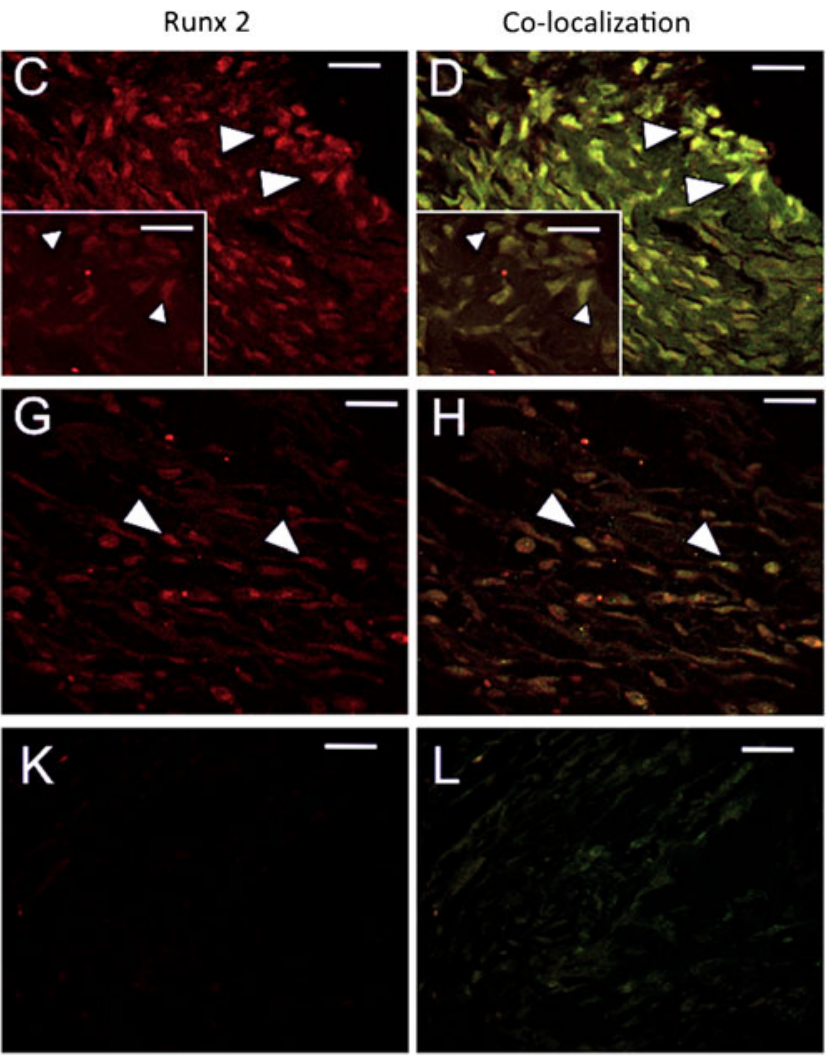

Fig. 4 Co-localization of Snail2 and Runx2 in a high-grade tibial osteosarcomas. H\&E stain $(A, E, I)$, immunolocalisation of Snail2 (B, $F, J)$, Runx $2(C, G, K)$ and co-localization $(D, H, L)$. High-grade tumours are shown $(A-H)$ and low-grade tumour $(I-L)$. Boxed area

consistent correlation between grade and Snail2 in more than one species. However, it also suggests that nuclear Snail2 is not a good correlate for metastasis in cats at least, as the overall incidence of this complication in this species is low.

Correlation between expression of Snail2 and tumour grade

The intensity of Snail2 immunostaining was scored based on four levels: negative (0), weakly (1), moderately (2) or

shows regions used for Snail2 and Runx 2 co-localization. In highgrade tumours, Snail2 and Runx2 co-localise (arrowheads in $D$ and $H$ ). Arrowheads indicate the same cells expressing both proteins. Inserts in $B-D$ are $\times 100$ magnification. Scale bar $=10 \mu \mathrm{m}$

strongly positive (3). A second score was employed for evaluating the extent of Snail2 expression. This was based on a three-point scale for distribution, with slides being scored as $0-15 \%$ (1), $16-30 \%$ (2) and $>30 \%$ (3) of cells stained. For each sample, a cumulative score was also generated by multiplying the intensity and distribution scores for Snail2 staining. This serves to expand the number of possible scores for any given tumour. Data have been summarized in Table 4. To determine whether any of

Table 2 Summary of clinical and histology data from canine cranial osteosarcoma

$O B$ osteoblastic, $C B$ chondroblastic, $F B$ fibroblastic

\begin{tabular}{lllllll}
\hline Case no. & Age & Sex & Breed & Tumour & Histology & Histology \\
\hline 1 & 14,0 & $\mathrm{M}$ & Bull terrier & Mandible & FB & High grade \\
2 & 10,6 & $\mathrm{~F}$ & Lurcher & Premolar tooth & OB & Low grade \\
3 & 10,0 & $\mathrm{M}$ & GSD & Mandible & CB & Low grade \\
4 & 8,0 & $\mathrm{M}$ & Pointer & Mandible & OB & Intermediate \\
5 & 13,9 & $\mathrm{M}$ & Retriever & Oral cavity & OB & Low grade \\
6 & 12,0 & $\mathrm{M}$ & Chow cross & Oral gingiva & OB & Intermediate \\
7 & 15,11 & $\mathrm{M}$ & Retriever & Oral mass & OB & Low grade \\
8 & 14,0 & $\mathrm{~F}$ & SBT & Mandible & OB & High grade \\
9 & 10,0 & $\mathrm{~F}$ & Spaniel & Oral mucosa & OB & High grade \\
10 & 9,0 & $\mathrm{~F}$ & Labrador & Oral mass & OB & High grade \\
\hline
\end{tabular}



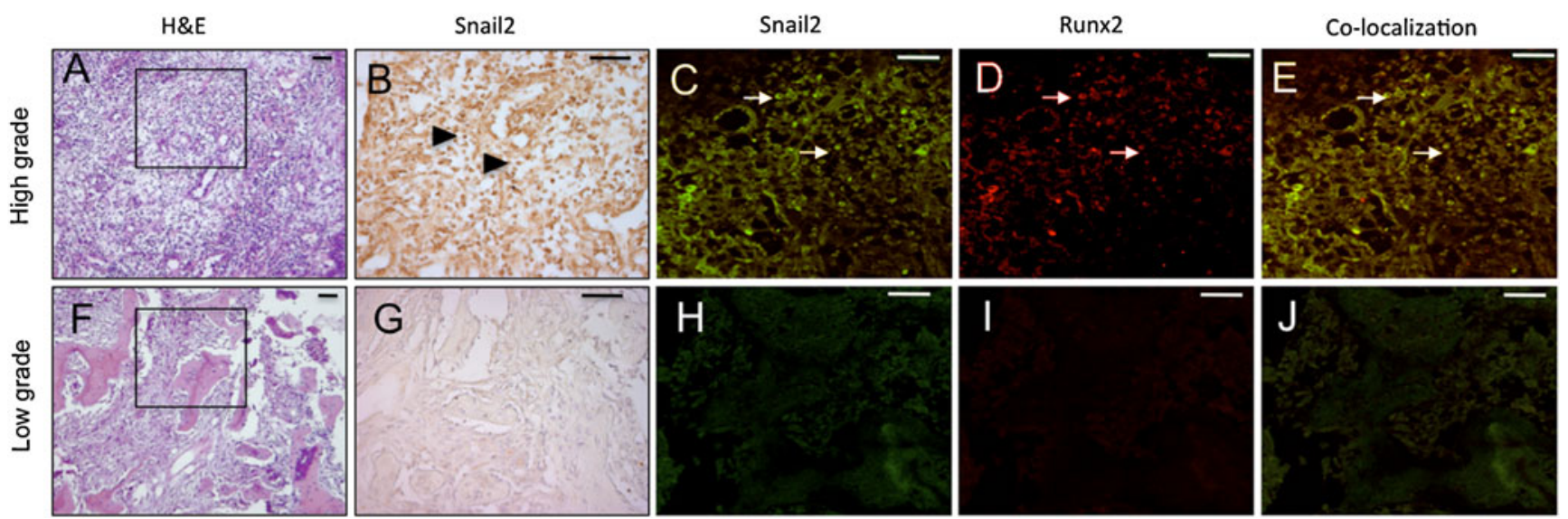

Fig. 5 Co-localization of Snail2 and Runx2 in a high-grade cranial osteosarcoma. H\&E stain $(A, F)$, immunolocalisation of Snail2 $(B, C$, $G, H)$, Runx $2(D, I)$ and co-localization $(E, J)$. High-grade $(A-E)$ and low-grade $(F-J)$ tumours shown. In high-grade tumours, Snail2 and

these scores could be used as a prognostic indicator of severity, we tested the correlation between tumour grade and each score. This showed that both increased intensity for Snail2 and cumulative score are associated with severity of tumour $(\mathrm{OR}=11.39,95 \%$ CI $1.62-80.0, p=0.014$; $\mathrm{OR}=$ $2.57,95 \%$ CI 1.16-5.69, $p=0.020$, respectively). However, distribution scores for Snail2 showed no statistically significant correlation with different tumour grade categories $(\mathrm{OR}=3.94,95 \%$ CI $0.85-18.2, p=0.08)$. We also examined the association between tumour grade and Snail2 intensity in canine cranial and feline appendicular osteosarcomas (Tables 5 and 6). However, there was no significant association between tumour grade and Snail2 intensity in cranial osteosarcoma ( $p=0.26$; see supplementary Table 2 ). An ordinal logistic regression was fitted for the feline data set; however, the algorithm failed to converge due to quasicomplete separation as a result of the small group sizes (See supplementary Table 3). When Snail2 intensity increases the tumour grade also increases (Kendall tau- $b=0.757, p<0.001$; see Table 6).

Table 3 Summary of clinical and histology data from feline long bone osteosarcoma

$O B$ osteoblastic, $C B$ chondroblastic, $F B$ fibroblastic
Runx 2 co-localise (arrows in $E$ ). Arrows in $C$ and $D$ indicate the same cells express both proteins. Low-grade tumours express little Snail2 or Runx2. Boxed area shows regions used for Snail2 and Runx2 colocalization. Scale bars $=10 \mu \mathrm{m}$

\section{Discussion}

Osteosarcomas are aggressive tumours that show a wide range of histological variants and cellular differentiation states. In canines and also humans, approximately $77 \%$ of osteosarcomas occur in the appendicular skeleton [12]. The site of origin of a tumour is an important factor that largely determines its characteristics. Typically tumours originating in the appendicular skeleton demonstrate a much more aggressive behaviour than those from other sites [19]. Advances in clinical management of osteosarcoma have been made; however, our understanding of the molecular pathogenesis of this disease is still rather limited. To date, there have been a limited number of prognostic markers identified that could be used in the clinic to identify patients with either a favourable or poor prognosis.

Two molecules that have been linked to metastasis in osteosarcoma are c-met and ezrin. C-met, the receptor for the hepatocyte growth factor, was found to be upregulated in primary canine osteosarcomas (including metastases)

\begin{tabular}{|c|c|c|c|c|c|c|c|}
\hline & \\
\hline & 1 & 9,4 & M & DLH & Femur & OB & Intermediate \\
\hline & 2 & 3,0 & M & DSH & Proximal tibia & OB & High grade \\
\hline & 3 & & $\mathrm{~F}$ & DLH & Stifle & OB & Low grade \\
\hline & 4 & 12,0 & $\mathrm{~F}$ & DSH & Femur & FB & High grade \\
\hline & 5 & 12,0 & $\mathrm{~F}$ & DLH & Distal femur & FB & High grade \\
\hline & 6 & 8,0 & M & DSH & Distal femur & $\mathrm{CB}$ & High grade \\
\hline & 7 & 14,0 & M & DSH & Humerus & OB & Low grade \\
\hline & 8 & 12,0 & $\mathrm{~F}$ & DSH & Tibia & OB & High grade \\
\hline & 9 & 9,6 & $\mathrm{~F}$ & DSH & Femur & FB & High grade \\
\hline $\begin{array}{l}O B \text { osteoblastic, } C B \text { chondro- } \\
\text { blastic, } F B \text { fibroblastic }\end{array}$ & 10 & 11,7 & M & DLH & Femur & OB & Intermediate \\
\hline
\end{tabular}




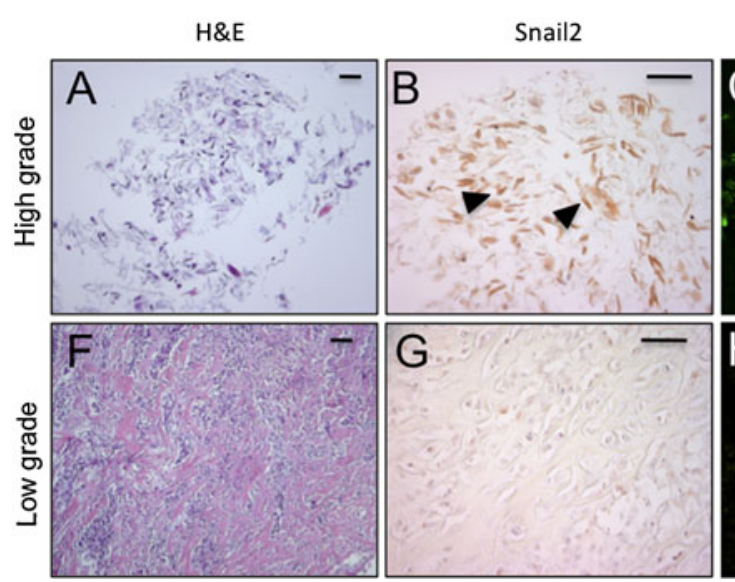

Fig. 6 Co-localization of Snail2 and Runx2 in a high-grade feline long bone osteosarcoma. H\&E stain $(A, F)$, immunolocalisation of Snail2 $(B, C, G, H)$, Runx $2(D, I)$ and co-localization $(E, J)$. Highgrade $(A-E)$ and low-grade $(F-J)$ tumours shown. Arrows in $C$ and $D$

[21], and inhibition of its function was able to limit invasion of canine osteosarcoma cells [22]. Ezrin, a cytoskeletal linker protein of the ERM family, has also been found in both canine and human osteosarcoma [23,24] and to be correlated with metastasis and poor clinical outcome.

There are, however, other genes that may be indicative of a more malignant phenotype, for example, pRB, p53 and
Snail 2
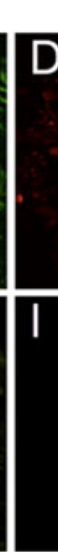

Co-localization
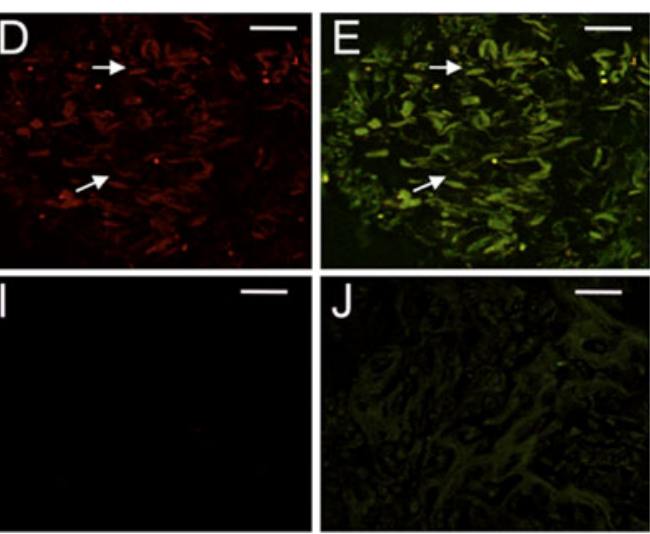

indicate the same cells express both proteins. Low-grade tumours express little Snail2 or Runx2. Boxed area shows regions used for Snail2 and Runx2 co-localization. Scale bar $=10 \mu \mathrm{m}$

the proto-oncogene c-fos [25] that are expressed in osteosarcoma. To date, mouse models of osteosarcoma have been developed, for example, pRb and p53 inactivation or c-fos activation, to aid in the study of osteosarcoma biology. These models do indeed produce tumours that contain neoplastic bone; however, it is unlikely that any of them truly recapitulates the human disease. They were all

Table 4 Relationship between Snail2 expression and tumour grade in canine long bone osteosarcoma

\begin{tabular}{|c|c|c|c|c|c|c|c|}
\hline \multirow[t]{2}{*}{ Case no. } & \multirow[t]{2}{*}{ Tumour grade } & \multicolumn{4}{|c|}{$\begin{array}{l}\text { Snail2 staining } \\
\text { intensity }\end{array}$} & \multirow{2}{*}{$\begin{array}{l}\text { Distribution of staining } \\
1(0-15 \%), 2(16-30 \%) \text {, } \\
3(>30 \%)\end{array}$} & \multirow{2}{*}{$\begin{array}{l}\text { Cumulative score } \\
\text { Intensity } \times \\
\text { distribution }\end{array}$} \\
\hline & & 0 & 1 & 2 & 3 & & \\
\hline 2 & High grade & & & & $\checkmark$ & 2 & 6 \\
\hline 3 & High grade & & & & $\checkmark$ & 3 & 9 \\
\hline 4 & High grade & & & $\checkmark$ & & 2 & 4 \\
\hline 5 & High grade & & & & $\checkmark$ & 3 & 9 \\
\hline 6 & High grade & & & $\checkmark$ & & 2 & 4 \\
\hline 8 & High grade & & & $\checkmark$ & & 1 & 2 \\
\hline 11 & High grade & & & $\checkmark$ & & 2 & 4 \\
\hline 12 & High grade & & $\checkmark$ & & & 1 & 1 \\
\hline 13 & High grade & & & $\checkmark$ & & 3 & 6 \\
\hline 16 & High grade & & & $\checkmark$ & & 2 & 4 \\
\hline 20 & High grade & & & $\checkmark$ & & 2 & 4 \\
\hline 1 & Intermediate & & $\checkmark$ & & & 2 & 2 \\
\hline 7 & Intermediate & & & $\checkmark$ & & 1 & 2 \\
\hline 14 & Intermediate & & & $\checkmark$ & & 2 & 4 \\
\hline 15 & Intermediate & & & $\checkmark$ & & 2 & 4 \\
\hline 18 & Intermediate & & $\checkmark$ & & & 1 & 1 \\
\hline 9 & Low grade & & $\checkmark$ & & & 2 & 2 \\
\hline 10 & Low grade & & $\checkmark$ & & & 1 & 1 \\
\hline 17 & Low grade & & & $\checkmark$ & & 1 & 2 \\
\hline 19 & Low grade & & $\checkmark$ & & & 2 & 2 \\
\hline
\end{tabular}


Table 5 Relationship between Snail2 expression and tumour grade in canine cranial osteosarcoma

\begin{tabular}{|c|c|c|c|c|c|c|c|}
\hline \multirow[t]{2}{*}{ Case no. } & \multirow[t]{2}{*}{ Tumour grade } & \multicolumn{4}{|c|}{$\begin{array}{l}\text { Snail2 staining } \\
\text { intensity }\end{array}$} & \multirow{2}{*}{$\begin{array}{l}\text { Distribution of staining } \\
1(0-15 \%), 2(16-30 \%), \\
3(>30 \%)\end{array}$} & \multirow{2}{*}{$\begin{array}{l}\text { Cumulative score } \\
\text { Intensity } \times \\
\text { distribution }\end{array}$} \\
\hline & & 0 & 1 & 2 & 3 & & \\
\hline 1 & High grade & & & & $\checkmark$ & 2 & 6 \\
\hline 8 & High grade & $\checkmark$ & & & & 1 & 0 \\
\hline 9 & High grade & & $\checkmark$ & & & 2 & 2 \\
\hline 10 & High grade & $\checkmark$ & & & & 1 & 0 \\
\hline 4 & Intermediate & & & & $\checkmark$ & 2 & 4 \\
\hline 6 & Intermediate & & $\checkmark$ & & & 1 & 1 \\
\hline 2 & Low grade & $\checkmark$ & & & & 1 & 0 \\
\hline 3 & Low grade & $\checkmark$ & & & & 1 & 0 \\
\hline 5 & Low grade & $\checkmark$ & & & & 1 & 0 \\
\hline 7 & Low grade & $\checkmark$ & & & & 1 & 0 \\
\hline
\end{tabular}

derived by a monogenetic event and thus may not sufficiently represent the heterogeneity of osteosarcoma. These models therefore may be inappropriate for studying the biology of these tumours and more importantly for testing therapies designed for human osteosarcoma. The similarity between canine and human osteosarcoma, coupled with the relatively higher number of canine cases, may make this a more apt model for studying osteosarcoma biology and the design and testing of therapies for human osteosarcoma.

In this study, we describe the expression of the transcription factor Snail2 in spontaneously occurring models of osteosarcoma, the first time this protein has been identified in this type of tumour in any species. Furthermore, we showed by immunohistochemistry that there was a positive correlation between levels of Snail2 expression and grade of tumour, suggesting that Snail2 could be another predictor of clinical outcome in cases of osteosarcoma.

Table 6 Relationship between Snail2 expression and tumour grade in feline long bone osteosarcoma
In epithelial tumours, Snail2 is an established marker of malignancy contributing to both the onset and progression of the disease [7]. The best characterised function of Snail2 in epithelial tumours is its ability to promote EMT, which is achieved in part by directly downregulating the cellular adhesion molecule E-cadherin and thus promoting cell migration [1].

Since osteosarcomas are tumours of mesenchymal origin [9], there is no need for cells to undergo EMT; therefore, Snail2 must be regulating other processes in this tumour type. However, the precise role that Snail2 plays in these mesenchymal tumours is yet to be defined. During embryonic development, Snail2 is expressed in some mesenchymally derived cells, for example, in proliferating chondrocytes and osteoblast precursors in endochondral bone [5].

Some of the functions of Snail2 in osteoblasts have recently been identified. In this instance, Snail2 has been shown to bind to the promoter of Runx2 and promote transcription [6]. Since Runx2 is a key regulator of the commitment of

\begin{tabular}{|c|c|c|c|c|c|c|c|}
\hline \multirow[t]{2}{*}{ Case no. } & \multirow[t]{2}{*}{ Tumour grade } & \multicolumn{4}{|c|}{$\begin{array}{l}\text { Snail2 staining } \\
\text { intensity }\end{array}$} & \multirow{2}{*}{$\begin{array}{l}\text { Distribution of staining } \\
1(0-25 \%), 2(26-50 \%) \text {, } \\
3(>50 \%)\end{array}$} & \multirow{2}{*}{$\begin{array}{l}\text { Cumulative score } \\
\text { Intensity } \times \\
\text { distribution }\end{array}$} \\
\hline & & 0 & 1 & 2 & 3 & & \\
\hline 2 & High grade & & & & $\checkmark$ & 2 & 6 \\
\hline 4 & High grade & & & $\checkmark$ & & 1 & 2 \\
\hline 5 & High grade & & & & $\checkmark$ & 1 & 3 \\
\hline 6 & High grade & & & & $\checkmark$ & 2 & 6 \\
\hline 8 & High grade & & $\checkmark$ & & & 1 & 1 \\
\hline 9 & High grade & & & $\checkmark$ & & 1 & 2 \\
\hline 1 & Intermediate & $\checkmark$ & & & & 1 & 1 \\
\hline 10 & Intermediate & $\checkmark$ & & & & 1 & 0 \\
\hline 3 & Low grade & $\checkmark$ & & & & 1 & 0 \\
\hline 7 & Low grade & & & $\checkmark$ & & 1 & 0 \\
\hline
\end{tabular}


mesenchymal cells to the osteoblastic lineage [26], Snail2 therefore promotes osteoblastic differentiation. It should be noted this is in marked contrast to the relationship between Snaill and Runx2, where Snail1 represses Runx2 and there is an inverse correlation in their expression [17]. This suggests that Snail2 and Snaill have opposing roles in osteoblast differentiation. This is a rare departure from the norm, where Snaill and Snail2 have similar or additive functions, and suggests that Snail transcription factors can have more complex roles than previously thought.

In our study, both Snail2 and Runx2 were present in osteoblastic osteosarcoma subtypes and in many cases were co-expressed. This therefore suggests that increased Snail2 expression upregulates Runx2 in osteoblastic osteosarcomas. The presence of Runx 2 in these tumours shows that they contain cells with the characteristics of osteoblastic progenitor cells. This is in agreement with other studies where osteosarcomas were shown to contain cells that have become trapped in the early stages of differentiation and that they remain refractory to osteogenic stimuli [27]. It could therefore be that maintained Snail2 and Runx2 expression results in an inability of these cells to differentiate fully, trapping them in preosteoblastic proliferative phenotype. Alternatively, it may be that Snail2 and Runx2 are expressed purely as a consequence of cells remaining in a preosteoblastic state (see Fig. 7). While we did not find an association between Snail2 and Runx2 in chondroblastic or fibroblastic osteosarcomas, we note that the numbers of these tumours examined were low.

It may be possible that Snail2 has other functions in osteosarcoma. One such function could be to decrease cellcell adhesion and therefore promote cellular migration producing a more aggressive tumour. Indeed, in canine appendicular long bone tumours, which we have shown to have high levels of Snail2, metastasis occurs in $80-90 \%$ of cases. We therefore went on examine Snail2 in canine osteosarcomas of the skull, which are rarer and have a lower frequency of metastasis [19]. In these cases, it was evident that Snail2 expression was present in much lower proportion of cases. This implies that Snail 2 could be involved in regulating cell migration. Alternatively, the different rates of metastasis may be purely due to the different origins of these tumours. It may be that neural crest-derived cells that give rise to cranial tumours are inherently less able to metastasize.
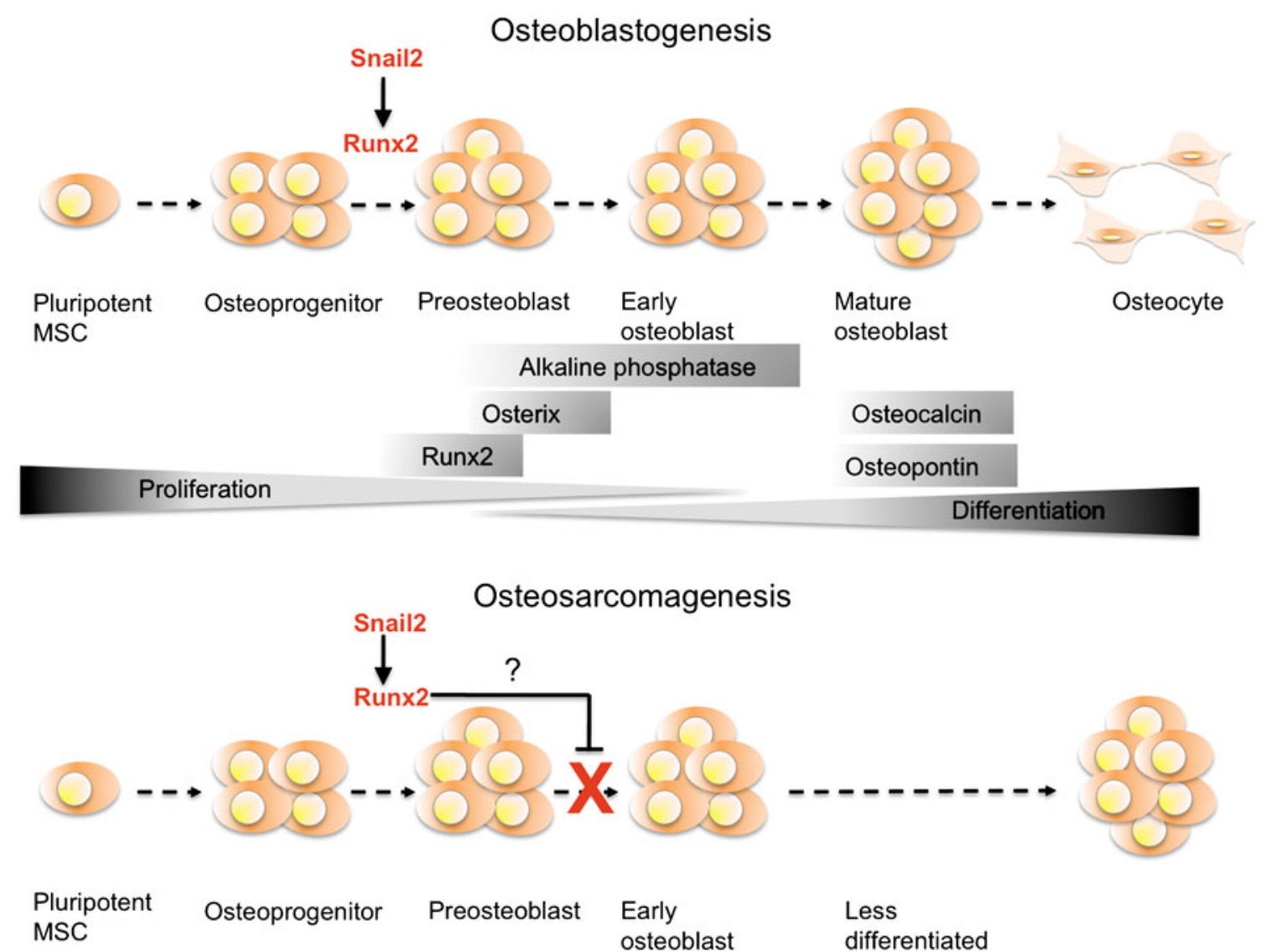

Fig. 7 A model for Snail2 and Runx2 activity in development of osteosarcoma. Upper panel: a diagrammatic representation of the function of Snail2 and Runx2 during normal bone differentiation. Snail2 promotes Runx2 expression and the progression of osteopro-

genitors to pre-osteoblasts. Lower panel: a model for the function of Snail2 and Runx2 in osteosarcoma. The increased levels of Snail2 keep Runx2 expression equally high. This prevents progression of cells to subsequent early and mature osteoblast states 
To further investigate whether Snail2 expression could be linked with metastasis, we analysed a series of feline osteosarcomas from appendicular long bone. In these cases, metastasis is known to occur in approximately $5-10 \%$ of animals compared with $80-90 \%$ in canines [20]. We would therefore expect lower levels of Snail2 in these cases if it is a regulator of metastasis. Our data show that in some of the feline cases, high levels of Snail2 were present and therefore Snail2 may not be associated with metastasis in felines. This study is, however, limited by the lack of clinical follow-up information, and we do not know whether the individual feline cases with high levels of Snail2 developed metastases in the future. It could therefore be possible that Snail2 promotes metastasis in felines; however, we currently lack the clinical data to investigate this. Further analysis of the role of Snail2 and Runx2 in cell-cell adhesion and in promoting cellular migration of osteosarcoma cells in other species including humans would allow us to confirm association of Snail2 with metastasis. Alternatively, there may be other explanations for why canine osteosarcomas are more metastatic than those in felines. We know that tumour development and metastasis are multifactorial events; therefore, it may be that in canines these tumours are genetically more prone to the events that induce metastasis and expression of Snail2 is a single event along this pathway.

In conclusion, Snail2 is statistically correlated with tumour severity in appendicular canine osteosarcomas. Since canine and human osteosarcomas are genetically very similar, this raises the possibility that Snail2 could be used as a prognostic marker in cases of human appendicular osteosarcoma.

Acknowledgements This study has been funded by a Cadogan studentship at the Royal Veterinary College and the Wellcome Trust (JP/ IM). We would like to thank Elaine Shervill for sectioning of tumours; Dr. Andy Sunters, Fahad Mahmood and Biggy Simbi for help with Western blots and Dr Ruby Chang for her expertise with statistical analysis.

\section{Conflicts of interest None}

Open Access This article is distributed under the terms of the Creative Commons Attribution Noncommercial License which permits any noncommercial use, distribution, and reproduction in any medium, provided the original author(s) and source are credited.

\section{References}

1. Nieto MA. The snail superfamily of zinc-finger transcription factors. Nat Rev Mol Cell Biol. 2002;3:155-66.

2. Hemavathy K, Ashraf SI, Ip YT. Snail/slug family of repressors: slowly going into the fast lane of development and cancer. Gene. 2000;257:1-12.

3. Del Barrio MG, Nieto MA. Relative expression of slug, rhob, and hnk-1 in the cranial neural crest of the early chicken embryo. Dev Dyn. 2004;229:136-9.

4. Xu J, Lamouille S, Derynck R. Tgf-beta-induced epithelial to mesenchymal transition. Cell Res. 2009;19:156-72.
5. Oram KF, Carver EA, Gridley T. Slug expression during organogenesis in mice. Anat Rec A Discov Mol Cell Evol Biol. 2003;271:189-91.

6. Lambertini E, Lisignoli G, Torreggiani E, Manferdini C, Gabusi E, Franceschetti T, et al. Slug gene expression supports human osteoblast maturation. Cell Mol Life Sci. 2009;66:3641-53.

7. Alves CC, Carneiro F, Hoefler H, Becker KF. Role of the epithelial-mesenchymal transition regulator slug in primary human cancers. Front Biosci. 2009;14:3035-50.

8. Weinberg RA. The biology of cancer. New York: Garland Science; 2007.

9. Mueller F, Fuchs B, Kaser-Hotz B. Comparative biology of human and canine osteosarcoma. Anticancer Res. 2007;27:155-64.

10. Gorlick R. Current concepts on the molecular biology of osteosarcoma. Cancer Treat Res. 2010;152:467-78.

11. Gorlick R, Khanna C. Osteosarcoma. J Bone Miner Res. 2010;25:683-91.

12. Selvarajah GT, Kirpensteijn J. Prognostic and predictive biomarkers of canine osteosarcoma. Vet J. 2010;185:28-35.

13. Slayter MV, Boosinger TR, Pool RR. Histological classification of bone and joint tumors of domestic animals (WHO International Classification of Tumors of Domestic Animals). American Registry of Pathology; 1994.

14. Armstrong VJ, Muzylak M, Sunters A, Zaman G, Saxon LK, Price JS, et al. Wnt/beta-catenin signaling is a component of osteoblastic bone cell early responses to load-bearing and requires estrogen receptor alpha. J Biol Chem. 2007;282:20715-27.

15. Loukopoulos P, Robinson WF. Clinicopathological relevance of tumour grading in canine osteosarcoma. J Comp Pathol. 2007; $136: 65-73$.

16. Tang N, Song WX, Luo J, Haydon RC, He TC. Osteosarcoma development and stem cell differentiation. Clin Orthop Relat Res. 2008;466:2114-30.

17. de Frutos CA, Dacquin R, Vega S, Jurdic P, Machuca-Gayet I, Nieto MA. Snail1 controls bone mass by regulating runx2 and vdr expression during osteoblast differentiation. EMBO J. 2009;28:68696.

18. Hemavathy K, Guru SC, Harris J, Chen JD, Ip YT. Human slug is a repressor that localizes to sites of active transcription. Mol Cell Biol. 2000;20:5087-95.

19. Chun R, de Lorimier LP. Update on the biology and management of canine osteosarcoma. Vet Clin North Am Small Anim Pract. 2003;33:491-516. vi.

20. Dimopoulou M, Kirpensteijn J, Moens H, Kik M. Histologic prognosticators in feline osteosarcoma: a comparison with phenotypically similar canine osteosarcoma. Vet Surg. 2008;37:466-71.

21. Fieten H, Spee B, Ijzer J, Kik MJ, Penning LC, Kirpensteijn J. Expression of hepatocyte growth factor and the proto-oncogenic receptor c-met in canine osteosarcoma. Vet Pathol. 2009;46:869-77.

22. De Maria R, Miretti S, Iussich S, Olivero M, Morello E, Bertotti A, et al. Met oncogene activation qualifies spontaneous canine osteosarcoma as a suitable pre-clinical model of human osteosarcoma. J Pathol. 2009;218:399-408.

23. Hunter KW. Ezrin, a key component in tumor metastasis. Trends Mol Med. 2004;10:201-4.

24. Khanna C, Wan X, Bose S, Cassaday R, Olomu O, Mendoza A, et al. The membrane-cytoskeleton linker ezrin is necessary for osteosarcoma metastasis. Nat Med. 2004;10:182-6.

25. Ragland BD, Bell WC, Lopez RR, Siegal GP. Cytogenetics and molecular biology of osteosarcoma. Lab Invest. 2002;82:365-73.

26. Komori T. Regulation of skeletal development by the runx family of transcription factors. J Cell Biochem. 2005;95:445-53.

27. Haydon RC, Luu HH, He TC. Osteosarcoma and osteoblastic differentiation: a new perspective on oncogenesis. Clin Orthop Relat Res. 2007;454:237-46. 